\title{
Rheumatoid arthritis, bone and drugs: a dangerous interweave
}

\author{
Salvatore Minisola 지 , Jessica Pepe, Cristiana Cipriani
}

Rheumatoid arthritis (RA) is a chronic autoimmune inflammatory condition that leads, per se, to bone loss by three main mechanisms. The first one is represented by bone erosions around the involved joints. Second, there is focal subchondral bone loss owing to increased osteoclast activity. Finally, systemic inflammation causes universal bone loss, mainly manifesting at the axial skeleton (ie, vertebral bodies and hip). ${ }^{1}$ On the top of this, physical inactivity, also related to lower muscle mass and sarcopenia, further exacerbates bone loss. As a consequence, osteoporosis can be detected in $30 \%-50 \%$ of patients with RA, also depending on age and sex; prevalence and fracture risk both raise with disease duration and seropositivity. ${ }^{2}$ The importance of $\mathrm{RA}$ in determining both local and systemic osteoporosis is emphasised by the fact that RAis an input variable, as independent risk factor, in the calculation of future fracture risk by FRAX.

It is also well known that patients with RA consume a number of drugs to mainly counteract inflammation and pain. However, some of the drugs used have detrimental effects on skeletal tissue, reducing bone strength and ultimately leading to fractures.

About $1 \%$ of adults in Britain and USA receive long-term oral glucocorticoids mainly for the treatment of joint problems but also for digestive and cutaneous diseases. $^{34}$ The long-term or high doses of glucocorticoids use is associated with reduced skeletal strength; indeed, glucocorticoid induced osteoporosis is the most prevalent cause of secondary osteoporosis. Glucocorticoids exert their deleterious effects on bone by acting on the three main cell skeletal lines (osteoblasts, osteoclasts end osteocytes). Among the most important negative effects are: (1) a preferential differentiation of pluripotent precursor cells to adipocytes rather than osteoblasts; (2) a stimulation of the

Department of Clinical, Internal, Anaesthesiological and Cardiovascular Sciences, Sapienza University of Rome, Roma, Italy

Correspondence to Dr Salvatore Minisola, Department of Clinical, Internal, Anaesthesiological and Cardiovascular Sciences, Sapienza University of Rome, Roma 00185, Italy; salvatore.minisola@uniroma1.it receptor activator of nuclear factor- $k B$ (RANK)-RANK ligand (RANKL) production which at least initially increase bone resorption and (3) an increased apoptosis of osteocytes with changes in the physical and fluid characteristics of the surrounding territory. These negative effects at the cellular levels contribute to increase the risk of fracture, together with the effects on other organs such as muscle (steroid myopathy) and eye (increased intra-articular pressure and formation of posterior subcapsular cataracts). ${ }^{5}$

Proton pump inhibitors (PPI) are drugs widely prescribed in the world. When Food and Drug Administration approved its use as an 'over-the-counter' drug, there has been a skyrocketing increase in their consumption. Data in Europe mirrored this trend. ${ }^{6}$ However, PPIs are associated with a number of side effects among which an increased risk of fracture. Indeed, a number of meta-analyses showed a significantly increased risk of hip and vertebral fractures, with some of them also suggesting a dose-dependent relationship. ${ }^{78}$ However, while the biological mechanisms leading to fractures in patients taking glucocorticoids are well ascertained, the same is not true for PPI. Hypothetical mechanisms include a reduced intestinal calcium absorption related to the hypochloridria induced by the negative effects on $\mathrm{H}^{+}$/ $\mathrm{K}^{+}$ATPase activity. The absorption of other micronutrients important for skeletal health are also adversely affected by long-term PPI use; in particular, PPIs use may be associated with hypomagnesaemia and a dose-response between the PPIs use and development of hypomagnesaemia has been reported. ${ }^{9}$ Other putative mechanisms include an excessive histamine production driven by hypergastrinaemia activated enterochromaffin-like cells (possibly leading to enhanced osteoclastogenesis and bone resorption) together with vitamin $\mathrm{B}_{12}$ deficiency. The last one has been associated with an increased risk of falls and with an increased risk of fractures. ${ }^{10} 11$

In this context, Abtahi et $a l^{12}$ add important information with implications in clinical practice that should change our behaviour. In summary, they showed that patients with RA taking both GC and PPI have a 1.6-fold increased risk of osteoporotic fractures (hip, clinical vertebral, pelvis and ribs) compared with non-users but also with single users or oral GC or PPI. ${ }^{12}$ Merits of this investigation include the large database used together with a long observation period (ie, more than 9 years). However, there are also important weaknesses in addition to those listed by the authors. The most important is represented by the lack of full control for bone active drugs taken by the patients, also including calcium and vitamin D. They claim that these drugs 'were not considered in the main analysis because of the accompaniment of their prescription with those of oral glucocorticoids and we expect them to lie in the causal pathway of the intended associations of mediators'. However, they do not show any evidence of this assumption nor the use of these drugs in the past 6 months is a guarantee for continuous use during the whole observation period. In addition, and most importantly, their data are not from a randomised controlled study; therefore, confounding cannot be excluded. For example, the populations considered might differ in terms of other factors that predispose or prevent fractures, such as the frequency and type of contact with care health system, physician prescribing attitudes and so on.

Bearing in mind these limitations, the most important message coming from this paper is a call for a watchful behaviour by doctors taking care of patients with RA on concurrent GC and PPI treatment. Interestingly, this dangerous liaison has been highlighted in an almost contemporary paper by Miyano et $a l^{13}$ in patients with anti-neutrophil cytoplasmic antibody (ANCA)-associated vasculitis.

Abtahi and coworkers conclude that fracture risk assessment could be considered when a patient with RA is coprescribed oral glucocorticoids and PPIs. This approach that we would say 'must' be considered, does not preclude an integration with another approach represented by reducing or de-prescribing drugs when they are not needed or when their use is not fully supported by the evidence. This is particularly true, considering the significant number of drugs prescribed to patients with RA that have been associated with an increased risk of fractures. ${ }^{14}$

As far as glucocorticoids, the first step should be an attempt to minimise the use of oral glucocorticoids in terms of both dosage and duration. Then, there is clear evidence that the combination of glucocorticoids with non-steroidal anti-inflammatory drugs increase the risk of peptic ulcer disease, thus justifying the concomitant use of PPI. However, there is conflicting evidence 
about the risk of peptic ulcer disease in patients taking glucocorticoid monotherapy. In a nested case-control study of Medicaid patients, there was no increased risk of peptic ulcer disease at any dose or length of glucocorticoid treatment. ${ }^{15}$ Looking for example at figure 1 (considering the pharmacological history in the 6 months before), only in 2954 patients the use of PPIs seems justified in those already taking steroids (2309 patients taking NSAIDs, 409 Cox-2 selective inhibitors, 198 with gastrooesophageal reflux and 38 with peptic ulcer disease) leaving the remaining $30.5 \%$ coprescriptions not appropriate or at least debatable. On the other hand, inappropriateness is also present in those taking steroids alone (1202, 205, 94 and 15 patients respectively) and in majority of those taking PPIs alone. This last group, points once again on the long-standing debate on overcoming resistance on deprescribing PPI. ${ }^{16}$

In those taking PPIs, an alternative approach to circumvent low calcium absorption due to hypochloridria (with subsequent skeletal loss owing to secondary hyperparathyroidism) may be represented by the administration of calcium citrate. This is because calcium carbonate is not soluble in water thus needing adequate acid secretion for ideal absorption. It has also the advantage of an optimal absorption independent of food intake. ${ }^{17}$ Furthermore, alternative therapies (at least in the first instance) including antacids, alginates or histamine type- 2 receptor antagonists can be attempted.

In conclusion, the paper by Abtahi et $a l,{ }^{12}$ brings to the light an important issue focusing on multiple coprescription of drugs potentially detrimental for skeletal health in patients ith RA. This aspect is often disregarded in clinical practice not only by general practitioners but also in referral centres. Add another drug to protect bone is an option; a number of studies in general not specifically targeted to patients with $\mathrm{RA},{ }^{18}$ have shown satisfactory results in terms of bone mineral density increase and fracture risk reduction in patients taking oral glucocorticoids. ${ }^{19-22}$ However, also reconsidering drug prescription is a suitable alternative to follow. Our suggested approach to protect bone in patients with RA taking steroids in addition to multidrug therapy is summarised in box 1 .

\section{Handling editor Josef S Smolen}

Contributors Intellectual concept of the editorial and approval of the submitted version.

Funding The authors have not declared a specific grant for this research from any funding agency in the public, commercial or not-for-profit sectors.

Box 1 Skeletal protection in patients with rheumatoid arthritis on steroids and multidrug therapy

1. Reduce glucocorticoids dose and consider glucocorticoids sparing treatments.

2. Improve nutrition (protein, calcium and vitamin D).

3. Adopt a healthy lifestyle (avoid tobacco, alcohol and perform physical activities and weight-bearing exercises).

4. Evaluate the panel of drugs prescribed.

5. Deprescribe, if possible, or consider alternatives drugs not harmful to bone.

6. Consider prescription of bone active drugs.

7. Monitor and reassess when indicated.

Competing interests None declared.

Patient and public involvement Patients and/or the public were not involved in the design, or conduct, or reporting, or dissemination plans of this research.

Patient consent for publication Not required.

Provenance and peer review Commissioned; internally peer reviewed.

(c) Author(s) (or their employer(s)) 2021. No commercial re-use. See rights and permissions. Published by BMJ.

\section{Check for updates}

To cite Minisola S, Pepe J, Cipriani C. Ann Rheum Dis 2021;80:409-410.

Received 2 January 2021

Accepted 6 January 2021

Published Online First 17 January 2021

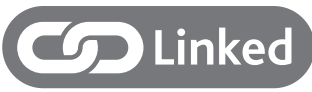

- http://dx.doi.org/10.1136/annrheumdis-2020218758

Ann Rheum Dis 2021;80:409-410.

doi:10.1136/annrheumdis-2020-219545

\section{ORCID iD}

Salvatore Minisola http://orcid.org/0000-0001-65250439

\section{REFERENCES}

1 Ashai S, Harvey NC. Rheumatoid arthritis and bone health. Clin Med 2020;20:565-7.

2 Hauser B, Riches PL, Wilson JF, et al. Prevalence and clinical prediction of osteoporosis in a contemporary cohort of patients with rheumatoid arthritis. Rheumatology 2014;53:1759-66.

3 Fardet L, Petersen I, Nazareth I. Prevalence of longterm oral glucocorticoid prescriptions in the UK over the past 20 years. Rheumatology 2011;50:1982-90.

4 Overman RA, Yeh J-Y, Deal CL. Prevalence of oral glucocorticoid usage in the United States: a general population perspective. Arthritis Care Res 2013;65:294-8

5 Chotiyarnwong P, McCloskey EV. Pathogenesis of glucocorticoid-induced osteoporosis and options for treatment. Nat Rev Endocrinol 2020;16:437-47.

6 Skjødt MK, Ostadahmadli Y, Abrahamsen B. Long term time trends in use of medications associated with risk of developing osteoporosis: nationwide data for Denmark from 1999 to 2016. Bone 2019:120:94-100

7 Zhou B, Huang Y, Li H, et al. Proton-Pump inhibitors and risk of fractures: an update meta-analysis. Osteoporos Int 2016;27:339-47.

8 Wei J, Chan AT, Zeng C, et al. Association between proton pump inhibitors use and risk of hip fracture: a general population-based cohort study. Bone 2020;139:115502.

9 Srinutta T, Chewcharat A, Takkavatakarn K, et al. Proton pump inhibitors and hypomagnesemia. Medicine 2019;98:e17788.

10 Kuschel BM, Laflamme L, Möller J. The risk of fall injury in relation to commonly prescribed medications among older people--a Swedish case-control study. Eur J Public Health 2015;25:527-32.

11 Lewis JR, Barre D, Zhu K, et al. Long-Term proton pump inhibitor therapy and falls and fractures in elderly women: a prospective cohort study. J Bone Miner Res 2014;29:2489-97.

12 Abtahi S, Driessen JHM, Burden AM. Concomitant use of oral glucocorticoids and proton pump inhibitors and risk of osteoporotic fractures among patients with rheumatoid arthritis: a population-based cohort study. Ann Rheum Dis 2021;80:423-31.

13 Miyano S, Michihata N, Sada K-E, et al. Comparison of fracture risk between proton pump inhibitors and histamine-2 receptor antagonists in ANCA-associated vasculitis patients: a nested case-control study. Rheumatology 2020;17:keaa594.

14 Ozen G, Pedro S, Wolfe F, et al. Medications associated with fracture risk in patients with rheumatoid arthritis. Ann Rheum Dis 2019;78:1041-7.

15 Piper JM, Ray WA, Daugherty JR, et al. Corticosteroid use and peptic ulcer disease: role of nonsteroidal anti-inflammatory drugs. Ann Intern Med 1991;114:735-40.

16 Lee TC, McDonald EG. Deprescribing proton pump inhibitors. JAMA Intern Med 2020;180:571-3.

17 Pepe J, Colangelo L, Biamonte F, et al. Diagnosis and management of hypocalcemia. Endocrine 2020:69:485-95.

18 Raterman HG, Lems WF. Pharmacological management of osteoporosis in rheumatoid arthritis patients: a review of the literature and practical guide. Drugs Aging 2019;36:1061-72.

19 Glüer C-C, Marin F, Ringe JD, et al. Comparative effects of teriparatide and risedronate in glucocorticoid-induced osteoporosis in men: 18-month results of the EuroGIOPs trial. J Bone Miner Res 2013;28:1355-68.

20 Geusens P, Marin F, Kendler DL, et al. Effects of teriparatide compared with risedronate on the risk of fractures in subgroups of postmenopausal women with severe osteoporosis: the VERO trial. J Bone Miner Res 2018;33:783-94.

21 Saag KG, Wagman RB, Geusens P, et al. Denosumab versus risedronate in glucocorticoid-induced osteoporosis: a multicentre, randomised, doubleblind, active-controlled, double-dummy, noninferiority study. Lancet Diabetes Endocrinol 2018;6:445-54.

22 Kendler DL, Marin F, Zerbini CAF, et al. Effects of teriparatide and risedronate on new fractures in post-menopausal women with severe osteoporosis (VERO): a multicentre, double-blind, doubledummy, randomised controlled trial. The Lancet 2018:391:230-40. 